\title{
Laser induced fluorescence of polycyclic aromatic hydrocarbons: An approach to gas standards
}

\author{
Patricia B. C. Forbes ${ }^{\mathrm{a}, \mathrm{b}^{*}}$, Andreas Trüe ${ }^{\mathrm{a}}$ and Egmont R. Rohwer ${ }^{\mathrm{b}}$ \\ ${ }^{a}$ Natural Resources and the Environment, Council for Scientific and Industrial \\ Research, PO Box 395, Pretoria, 0001, South Africa. \\ ${ }^{b}$ Department of Chemistry, Faculty of Natural and Agricultural Sciences, \\ University of Pretoria, Pretoria, 0002, South Africa. \\ * To whom correspondence should be addressed.
}

Ph: +27128413031

Fax: +27128412689

pforbes@csir.co.za

\section{ABSTRACT}

Reliable semi-quantitative gas standards were required for the development of a laser induced fluorescence (LIF) method for polycyclic aromatic hydrocarbon analysis, based on sampling of air onto multi-channel polydimethylsiloxane rubber traps. Easily constructed diffusion tubes provided naphthalene vapour at $\sim 2 \mathrm{ng} . \mathrm{s}^{-1}$. A gas chromatographic fraction collection method for loading less volatile PAHs onto the traps from a flame ionization detector outlet was developed and optimized. The accuracy of the method, which can be further optimised, was sufficient for initial LIF screening tests to flag samples exceeding threshold PAH levels for subsequent quantitative GC-MS analysis.

Keywords: Diffusion tubes; polydimethylsiloxane; gas standards; polycyclic aromatic hydrocarbons; gas chromatographic fraction collection. 


\section{INTRODUCTION}

Polycyclic aromatic hydrocarbons (PAHs) are air pollutants of environmental concern due to their toxicity and the effective monitoring of these compounds is important as they are ubiquitous environmental pollutants. In order to reduce analytical costs and thereby increase sampling coverage, we have developed a method which may serve as a screening tool in atmospheric PAH analysis with final confirmation (and quantitation) by gas chromatography-mass spectrometry (GC-MS). This method is based on the sampling of air onto multi-channel polydimethylsiloxane (PDMS) rubber traps (Ortner and Rohwer 1996), which contain 22 parallel, $0.3 \mathrm{~mm}$ i.d. PDMS tubes housed inside a quartz tube. Semi-quantitative analysis of these traps is achieved by laser induced fluorescence (LIF) utilizing a suitable excitation wavelength for the $\mathrm{PAH}(\mathrm{s})$ of interest and by quantifying the fluorescence emission thus obtained at defined wavelength(s) (Forbes and Rohwer 2009). Analytical methods have also been developed for the direct comprehensive analysis of the PDMS traps post LIF analysis by thermal desorption-gas chromatographymass spectroscopy (TD-GC-MS).

Sampling of analytes on the PDMS traps is achieved via sorption, where analytes interact with the trap according to gas chromatographic principles. It was therefore necessary to load known quantities of PAH standards onto the traps in a suitable manner, which allowed for the uniform distribution of the standard in cross section over each of the PDMS tubes, and preferably introduced the standard into the trap in the form of a plug. Although injection of a liquid standard onto a trap, followed by passing a fixed volume of nitrogen gas through the trap has been reported (Wauters et al. 2008), this method is not ideal for multichannel silicone rubber traps in the LIF application, as it is difficult to equally distribute a small volume of liquid over the 22 inlets of the silicone tubes. A convenient and cost effective alternative was therefore sought, which may find application in other spectroscopic studies particularly where pure solid standards are not available.

The generation of gas standards, as reviewed by Barratt (1981), Namieśnik (1984) and Naganowska-Nowak et al. (2005), may be broadly classified into static and dynamic methods. Static techniques involve the introduction of a specific amount of analyte into a known volume of diluent gas in a closed container, whilst with dynamic methods the analyte enters the diluent gas stream on a continuous basis (Naganowska-Nowak et al. 2005).

Static systems, such as gas cylinders containing standard mixtures, are used primarily for small volumes of mixtures and fairly high concentrations, but losses of components of 
the mixture to the wall of the container may occur, and these systems are usually not very portable and are very expensive. Dynamic systems can produce larger volumes, with surface losses being lower than in static systems due to the existence of equilibrium conditions after saturation of contact surfaces, and are thus more suitable for polar and reactive species (Barratt 1981).

Whilst diffusion tubes, which are a well established dynamic method for gas standard generation, were suitable for naphthalene, an alternative method was needed for the less volatile PAHs of interest, specifically phenanthrene and pyrene, where diffusion tubes would only yield pg.s ${ }^{-1}$ diffusion rates. A gas chromatographic fraction collection method was therefore developed for this purpose, where a fixed mass of analyte was transferred onto a sample trap placed on the flame ionization detector (FID) tip. Knowledge of the retention time of the analyte under the GC conditions employed allowed for the collection of the analyte only, and not the solvent or other impurities. A similar method has been used to collect individual components of beer (Lim ah Tock 2008) and milk (Naudé et al. 2009) aroma, in this case for organoleptic evaluation of such fractions. The practicality of this collection method is based on the negligible pressure drop of the open tubular structure of the trap, not influencing the flow rate through the GC column. It was necessary to optimize the experimental setup in order to minimize analyte loss (including possible losses due to breakthrough) and to maximize trapping efficiencies.

\section{EXPERIMENTAL}

\subsection{Naphthalene diffusion tube}

The diffusion tube assembly consisted of a glass vessel ( $4.5 \mathrm{~cm}$ long, $8 \mathrm{~mm}$ i.d.), which was attached to a glass capillary $(3.5 \mathrm{~cm}$ long, $6 \mathrm{~mm}$ i.d.) by means of a ground glass joint. Naphthalene (Fluka, $99.8 \%$ purity, GC grade) was placed inside the vessel, and the capillary was then positioned and the joint tightly closed. The diffusion tube was allowed to equilibrate under temperature controlled conditions, and was then weighed at various time intervals to $0.01 \mathrm{mg}$ (Mettler analytical balance).

The diffusion tube was placed in a glass diffusion chamber, which comprised two portions joined by a tightly sealed ground glass joint, as shown in Figure 1. This allowed for easy insertion and removal of the diffusion tube. The diffusion chamber consisted of both an inlet and outlet port for the diluent gas flow. Sufficient re-equilibration time was allowed after insertion of the diffusion tube into the diffusion chamber prior to usage of the device. A battery operated portable sampling pump (Gilair) was used to draw air at $\sim 500 \mathrm{~m} \ell \cdot \mathrm{min}^{-1}$ into the sample holder and then onto the silicone rubber trap. 
The laboratory room air was also sampled onto a trap for 5 and 60 min, respectively, at $477 \mathrm{~m} \ell \cdot \mathrm{min}^{-1}$ prior to analysis, in order to verify that all the naphthalene present on loaded traps originated from the diffusion tube.

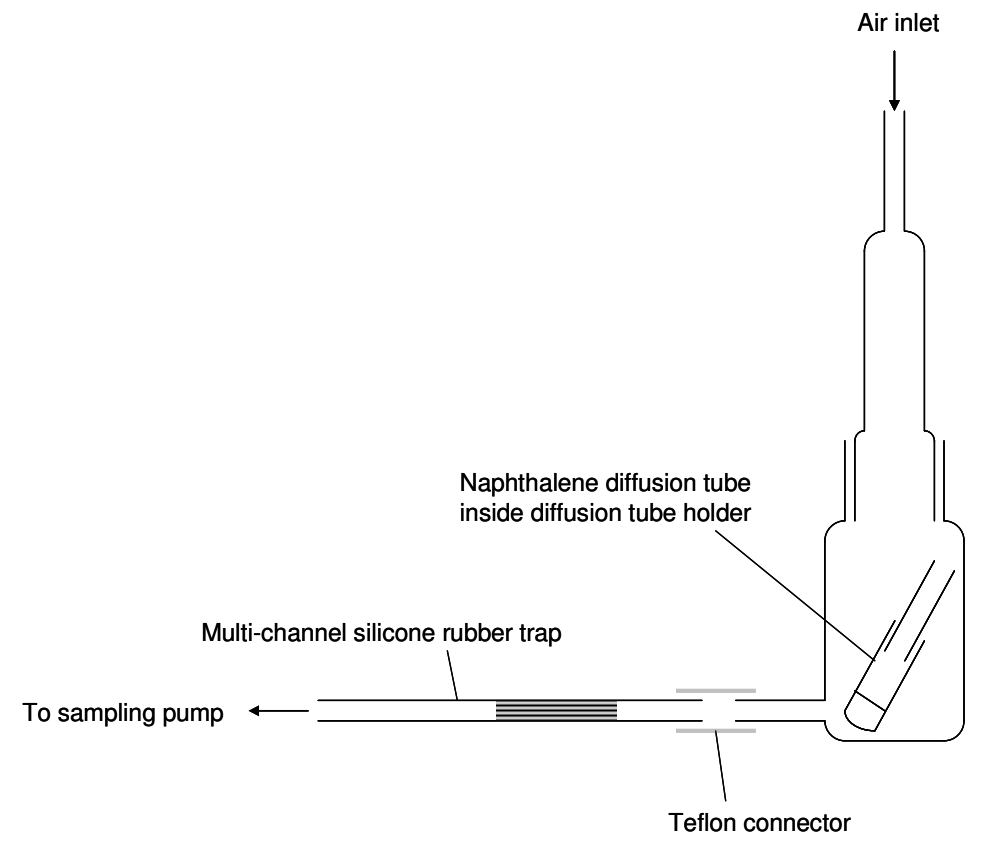

Figure 1: Use of the naphthalene diffusion tube to load a silicone rubber trap with gaseous phase standard.

The direction of air flow through the diffusion tube chamber was also reversed, in order to determine whether the flow regime was inducing turbulent conditions, which would impact on the rate of diffusion. A trap was repeatedly loaded for a period of $5 \mathrm{~min}$.

\subsection{PDMS trap loading via gas chromatographic fraction collection}

Liquid standards of the PAHs (naphthalene: Fluka, $99.8 \%$ purity, GC grade; phenanthrene, pyrene, fluorene and anthracene: Supelco, $99.9 \%, 96.6 \%, 98.6 \%$ and $99.0 \%$ purity respectively, GC grade) were prepared in toluene (Riedel de Haen, GC grade, $99.7 \%$ purity), and were manually injected onto an EQUITY ${ }^{\mathrm{TM}}-5$ fused silica capillary column (Supelco, $30 \mathrm{~m} \times 0.25 \mathrm{~mm} \times 0.25 \mu \mathrm{m}$ ) of an Agilent 7890A GC equipped with an FID, which was maintained at $250{ }^{\circ} \mathrm{C}$ and was not ignited. In order to minimize losses at the FID outlet, the top assembly of the detector as well as the collector/insulator were removed and the multi-channel silicone rubber trap was connected directly to the flame tip by means of a small piece of Teflon tubing and was manually pressed into position at the appropriate time during collection runs (Figure 2). 


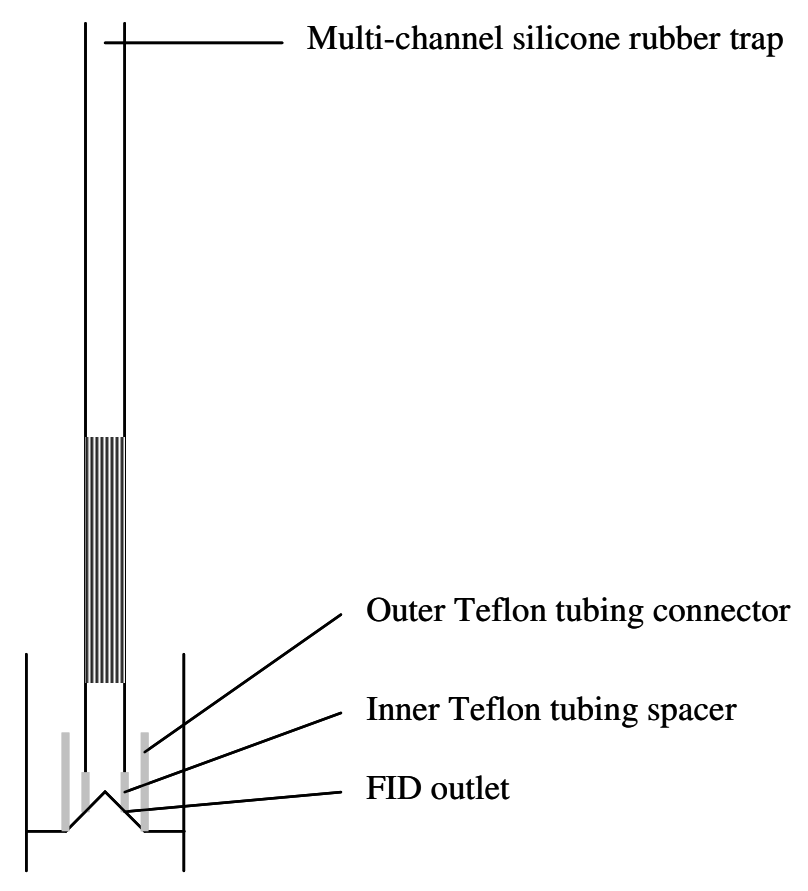

Figure 2: Experimental setup for the loading of PAHs onto multi-channel silicone rubber traps from the FID outlet. The outer Teflon connector fitted tightly over the trap and inner Teflon spacer. The igniter was disconnected and the top assembly of the FID as well as the collector and insulator were temporarily removed during the loading procedure.

The efficiency of loading was determined by comparison of TD-GC-MS peak areas obtained for FID loaded traps to that obtained for the direct injection of $1 \mu \ell$ of the mixed PAH standard (equivalent to $20 \mathrm{ng}$ of each PAH) initially onto an empty glass tube and later onto a sample trap, as specified for each experiment. The TD-GC-MS method detailed in section 2.3 was used.

\subsubsection{Optimization of the FID make-up flow rate}

$20 \mathrm{ng}$ of a mixed PAH standard (containing fluorene, anthracene, naphthalene, phenanthrene and pyrene) was injected as $1 \mu \ell$ using different FID make-up flow rates: 5; 10; 20; 50 and $100 \mathrm{~m} \ell \cdot \mathrm{min}^{-1}$. Initially, after a hold time of $10 \mathrm{~min}$ at $40^{\circ} \mathrm{C}$, the $\mathrm{GC}$ oven was ramped to $150{ }^{\circ} \mathrm{C}$ at $20{ }^{\circ} \mathrm{C} \cdot \mathrm{min}^{-1}$ and then to $300{ }^{\circ} \mathrm{C}$ at $30{ }^{\circ} \mathrm{C} \cdot \mathrm{min}^{-1}$. PAHs were collected onto a trap between 14 and $21 \mathrm{~min}$. In later experiments the hold time at $40^{\circ} \mathrm{C}$ was decreased to $1.5 \mathrm{~min}$, and the PAHs were collected from the FID outlet from 6 to 14.5 min after injection. The loaded traps were then analysed by TD-GC-MS using an empty glass tube for standard injections. 


\subsubsection{Repeatability}

\section{a) Initial experiments}

Four traps were loaded using the method described under section 2.2.1 (10 min GC hold time at $40{ }^{\circ} \mathrm{C}$ ). They were then end-capped and refrigerated overnight prior to TDGC-MS analysis using an empty glass tube for standard injections. This experiment was repeated $(n=4)$ with the PAH collection time from 15 to $21 \mathrm{~min}$.

\section{b) Optimised conditions}

The loading method was optimized by shortening the inner Teflon spacer between the FID tip and the trap from $1.4 \mathrm{~cm}$ to $4 \mathrm{~mm}$ in order to prevent losses by bringing the trap into closer contact with the flame tip thereby minimizing cooling and contact with the Teflon. The system was checked for leaks by measuring the flow rate of the gas exiting the trap to that of the detector make-up and column flow rate (using a Porter Instrument Company B495 flow meter), and no losses were evident.

The repeatability experiment was then redone with the shorter initial GC hold time and collection at the FID outlet from 6 to 14.5 min after injection $(n=7)$. Collection efficiencies were determined upon comparison with standards injected directly onto silicone rubber traps.

\subsubsection{Breakthrough experiments}

During the repeatability experiments described under section 2.2.2, one trap was loaded whilst a second trap was positioned in series (connected to the primary trap by means of a short piece of Teflon tubing). The secondary trap was then analysed by TD-GC-MS using an empty glass tube for standard injections. This experiment was also repeated after optimization of the collection time and the FID connection.

\subsubsection{Cooling of traps during loading}

In order to determine whether losses during loading could be decreased by cooling of the trap, nitrogen vapour from a small liquid nitrogen dewar was directed onto the portion of the trap where the silicone rubber tubes are located. Loading was performed with and without cooling $6 \mathrm{~min}$ after injection of $1 \mu \ell$ of $50 \mu \mathrm{g} \cdot \mathrm{m} \ell^{-1}$ naphthalene standard (i.e. the most volatile PAH was tested), with $n=2$, and the peak areas were compared to direct loading onto a silicone rubber trap.

\subsubsection{Stability of loaded traps}

In order to determine whether traps could be loaded with phenanthrene and pyrene the day before they were required as standards for analytical method development, five traps 
were loaded from the FID with phenanthrene and pyrene, respectively $(1 \mu \ell$ of 100 $\mu \mathrm{g} . \mathrm{m} \ell^{-1}$ ) according to the procedure described under section 2.2.1, with the shorter initial GC hold time at $40^{\circ} \mathrm{C}$ and with a three min hold time at $300^{\circ} \mathrm{C}$. The PAHs were collected onto the traps from 8 to $13 \mathrm{~min}$, after which they were analysed by TD-GC-MS. The results were compared to the analysis of five direct injections of a standard solution ( $1 \mu \ell$ of $100 \mu \mathrm{g} . \mathrm{m} \ell^{-1}$ ) onto a silicone rubber trap. Three traps were then loaded again with phenanthrene and pyrene, respectively. These traps were end-capped and stored in the refrigerator overnight. They were analysed the next day by TD-GC-MS, and the results were compared to that of direct injection onto a trap.

\subsection{TD-GC-MS analysis}

The loaded silicone rubber traps were thermally desorbed using a thermal desorber system (TDS) (Gerstel TDS 3) and the desorbed analytes were cryogenically focused via a cooled injection system (CIS). The traps were desorbed from $0{ }^{\circ} \mathrm{C}(0.2 \mathrm{~min})$ to $270{ }^{\circ} \mathrm{C}$ (10 min, or $2 \mathrm{~min}$ in the case of naphthalene analyses) at $120{ }^{\circ} \mathrm{C} \cdot \mathrm{min}^{-1}$ in the solvent vent mode (100 $\mathrm{m} \ell \cdot \mathrm{min}^{-1}$ until $0.2 \mathrm{~min}$ ). Cryo-focusing of the PAHs was achieved using liquid nitrogen at $-40{ }^{\circ} \mathrm{C}(0.5 \mathrm{~min})$ followed by rapid heating at $12{ }^{\circ} \mathrm{C} . \mathrm{s}^{-1}$ to $300{ }^{\circ} \mathrm{C}(10$ min, or $1 \mathrm{~min}$ in the case of naphthalene analyses). The GC-MS system was an Agilent GC 7890A coupled to a Hewlett Packard 5975 inert XL EI/CI mass selective detector (MSD). The GC inlet was in the split mode (10:1) with helium (Ultra High Purity, Afrox) as the inlet gas $\left(1.3 \mathrm{~m} \ell \cdot \mathrm{min}^{-1}\right.$ at $\left.40{ }^{\circ} \mathrm{C}\right)$. An Agilent HP5 $(30 \mathrm{~m}$ x $250 \mu \mathrm{m} \times 0.25$ $\mu \mathrm{m}) \mathrm{GC}$ column was used and the column head pressure was $68.8 \mathrm{kPa}$ using helium as the carrier gas. The GC oven was temperature programmed from $40{ }^{\circ} \mathrm{C}(1.5 \mathrm{~min})$ at 20 ${ }^{\circ} \mathrm{C}$. $\min ^{-1}$ to $150{ }^{\circ} \mathrm{C}(0 \mathrm{~min})$ and at $30{ }^{\circ} \mathrm{C} \cdot \mathrm{min}^{-1}$ to $300{ }^{\circ} \mathrm{C}(0.5 \mathrm{~min})$. The GC-MS transfer line was at $300{ }^{\circ} \mathrm{C}$, the mass scan range was $80-250$ atomic mass units (amu), the solvent delay $6.8 \mathrm{~min}$, and the electron multiplier voltage $\sim 1070 \mathrm{~V}$. The GC inlet was in the solvent vent mode $\left(100 \mathrm{~m} \ell \cdot \mathrm{min}^{-1}\right.$ until $\left.0.1 \mathrm{~min}\right)$ for the analysis of traps relating to sections 2.2.1, 2.2.2 and 2.2.3.

The limit of detection (LOD) of this TD-GC-MS method (CIS split of 10:1) was determined by the analysis of silicone rubber traps onto which $1 \mu \ell$ of PAH standards in toluene had been injected $(0 ; 0.2 ; 1 ; 2 ; 4 ; 8 ; 20 ; 40$ and $80 \mathrm{ng}$, respectively). 


\section{RESULTS AND DISCUSSION}

\subsection{Diffusion tubes}

The diffusion rate can be calculated from theoretical considerations:

$$
S=\frac{D M P A}{R T L} \ln \frac{P}{P-p} \quad \text { Equation } 1
$$

Where $S$ is the diffusion rate in $\mathrm{g} . \mathrm{s}^{-1} ; D$ is the diffusion coefficient $\left(\mathrm{cm}^{2} \cdot \mathrm{s}^{-1}\right) ; M$ is the molar mass of the compound $\left(\mathrm{g} \cdot \mathrm{mol}^{-1}\right) ; P$ is the pressure in the diffusion cell at the open end of the capillary (atm); $A$ is the cross-sectional area of the diffusion tube $\left(\mathrm{cm}^{2}\right) ; R$ is the molar gas constant $\left(\mathrm{m} \ell \cdot \mathrm{atm} \cdot \mathrm{mol}^{-1} \cdot \mathrm{K}^{-1}\right) ; T$ is the absolute temperature $(\mathrm{K}) ; L$ is the diffusion path length $(\mathrm{cm})$, and $p$ is the partial pressure of the diffusing vapour at temperature $T$ (atm).

The diffusion rate will only become constant once steady-state conditions have been reached. This can be estimated from the function (Altshuller and Cohen 1960):

$$
t>\frac{L^{2}}{2 D}
$$

Equation 2

Where $\mathrm{t}$ is the total time for steady-state conditions to be reached, in seconds.

Diffusion rates were thus calculated for the diffusion tubes at $20{ }^{\circ} \mathrm{C}$ (due to availability of vapour pressure and diffusion rates at this temperature), and $P=0.859 \mathrm{~atm}$ (average atmospheric pressure in Pretoria, South Africa, as obtained from the National Metrology Institute of South Africa 2007), with $R=82.06 \mathrm{~m} \ell$.atm. $\mathrm{mol}^{-1} \cdot \mathrm{K}^{-1}$, as presented in Table 1 .

Table 1: Theoretical diffusion rates, for $\mathrm{L}=3.5 \mathrm{~cm}$.

${ }^{\#}$ Obtained from the Danish Environmental Protection Agency (2006).

\begin{tabular}{|c|c|c|c|c|}
\hline Compound & $\mathbf{D}\left(\mathbf{c m}^{2} \cdot \mathbf{s}^{-1}\right)^{\#}$ & $\mathbf{M}\left(\mathbf{g} . \mathbf{m o l}^{-1}\right)$ & $\mathbf{p}(\mathbf{a t m})^{\#}$ & $\mathbf{S}\left(\mathbf{n g . s} \mathbf{s}^{-1}\right)$ \\
\hline Naphthalene & 0.069 & 128.2 & $1.03 \times 10^{-4}$ & 3.1 \\
\hline Phenanthrene & 0.058 & 178.2 & $1.58 \times 10^{-7}$ & $5.5 \times 10^{-3}$ \\
\hline Pyrene & 0.055 & 202.3 & $6.02 \times 10^{-9}$ & $2.2 \times 10^{-4}$ \\
\hline
\end{tabular}

From these calculations, it is evident that the theoretical diffusion rate for naphthalene was in the low ng.s ${ }^{-1}$ range, whilst that for the other, less volatile PAHs were much lower at pg. $\mathrm{s}^{-1}$ levels. The time required for steady-state conditions to be reached for these 
particular diffusion tube configurations was calculated to be in the range $1-2 \mathrm{~min}$ from equation 2 .

The gravimetric results obtained for the naphthalene diffusion tube over an extended period of $\sim 250$ days gave a diffusion rate of $1.9 \mathrm{ng} . \mathrm{s}^{-1}\left(\mathrm{r}^{2}=0.95\right)$. This is in good agreement with the theoretical diffusion rate of $3.1 \mathrm{ng} . \mathrm{s}^{-1}$ (refer to Table 1), even though there was a high mass ratio of the tube to its contents, and the diffusion path length was relatively short.

The LOD of the TD-GC-MS method was found to be $<0.5 \mathrm{ng}$ for each PAH, where the LOD was based on three times the standard deviation of the blank divided by the slope of the calibration graph for each PAH.

The laboratory air loading experiments yielded concentrations of $<1 \mathrm{ng}$ naphthalene after 5 min loading, and $1 \mathrm{ng}$ for a $60 \mathrm{~min}$ loading time. It was therefore deemed unnecessary to pre-clean laboratory air prior to its passage through the diffusion tube. This had the advantage of not increasing the back pressure through the system.

The chromatographic peak area for naphthalene increased by almost a factor of two when the reversed flow diffusion tube configuration was employed, which would indicate that the flow regime of the original configuration was more stable, and was therefore used for trap loading purposes. We ascribe the measured differences to the small length to diameter ratio of the diffusion tube, where even slight turbulence at the tube outlet can alter the diffusion rate of the analyte. This clearly means that wider and shorter diffusion tubes should not be used to increase the diffusion rate of the less volatile standards.

\subsection{PDMS trap loading via gas chromatographic fraction collection}

The results of the experiments relating to the optimization of the FID make-up flow rate are presented in Figure 3. It was decided to proceed with make-up flow rates of 50 $\mathrm{m} \ell \cdot \mathrm{min}^{-1}$, as this appeared to give the best recoveries in most cases, particularly for phenanthrene and pyrene, for which gas standard loading was most needed. 


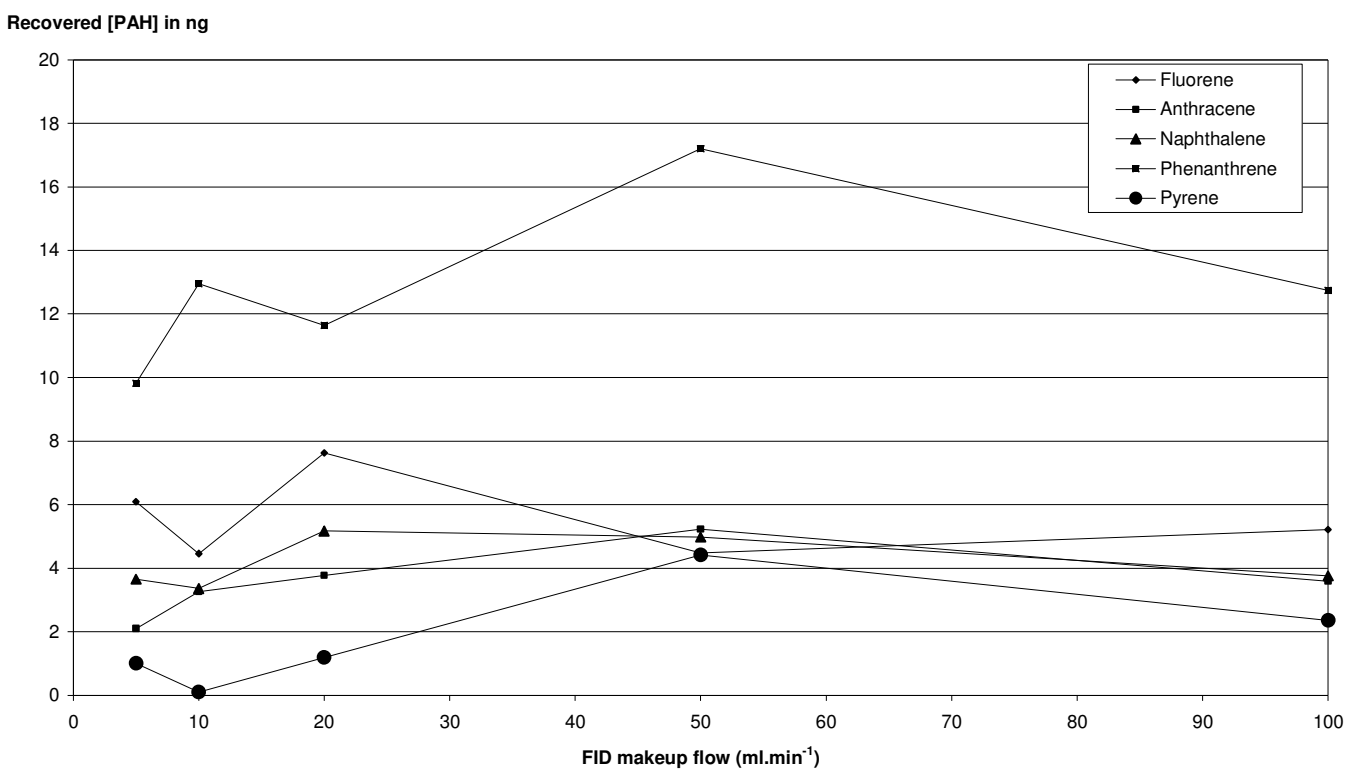

Figure 3: Recovery of $20 \mathrm{ng}$ of individual PAHs from multi-channel silicone rubber traps after gas chromatographic loading of a mixed standard using different FID make-up flow rates.

The results of the two sets of repeatability experiments are presented in Table 2. It is evident that the delayed collection time improved the repeatability for most of the PAHs, except phenanthrene and pyrene, but decreased the collection efficiency in most cases. Collection efficiencies and repeatability were therefore further optimized (refer to section 2.2.2 (b), and the results are presented in Table 3. It is evident that the recoveries had improved compared to the initial experiments, with the exception of phenanthrene, and that the repeatability had improved.

Table 2: Percentage recoveries for PAHs from $20 \mathrm{ng}$ gas chromatographic loading of a mixed standard, using different traps and different collection times.

\begin{tabular}{|l|c|c|}
\hline PAH & Average \% trapped & \% RSD \\
\hline $14-21$ min collection, $n=4$ & & \\
\hline Fluorene & 41 & 23 \\
\hline Anthracene & 21 & 41 \\
\hline Naphthalene & 35 & 38 \\
\hline Phenanthrene & 66 & 19 \\
\hline Pyrene & 52 & 41 \\
\hline $15-21$ min collection, $n=4$ & & 17 \\
\hline Fluorene & 27 & 22 \\
\hline Anthracene & 26 & \\
\hline
\end{tabular}




\begin{tabular}{|l|c|c|}
\hline Naphthalene & 28 & 24 \\
\hline Phenanthrene & 60 & 26 \\
\hline Pyrene & 39 & 40 \\
\hline
\end{tabular}

Table 3: Percentage recoveries for PAHs from gas chromatographic loading of a $20 \mathrm{ng}$ mixed standard under optimized conditions, using seven different traps.

\begin{tabular}{|c|c|c|}
\hline PAH & Average \% trapped & \% RSD \\
\hline 6-14.5 min collection, $n=7$ & & \\
\hline Fluorene & 61 & 12 \\
\hline Anthracene & 71 & 27 \\
\hline Naphthalene & 57 & 14 \\
\hline Phenanthrene & 52 & 18 \\
\hline Pyrene & 61 & 16 \\
\hline
\end{tabular}

From the peak areas obtained for the secondary trap for each PAH it was evident that breakthrough from the primary trap was minimal $(<1 \%)$. No significant breakthrough was also found when the experiment was repeated under optimized conditions, as the peak areas found on the secondary trap for each PAH were all less than $0.75 \%$ of the primary trap.

Cooling did not improve recoveries but actually had a negative impact and was not used in the final method.

The results of the experiments to establish the stability of loaded traps are summarized in Table 4. The recovery of FID loaded traps was $\sim 50 \%$ for both phenanthrene and pyrene when compared to direct injection, and the recovery did not decrease significantly when the loaded traps were stored overnight. The repeatability was generally good, with $\%$ RSDs of less than $15 \%$, with the exception of the stored phenanthrene traps, which had a $\%$ RSD of $29 \%$. This may have been improved by increasing the number of stored traps analysed.

Table 4: Effect of storage of GC loaded traps on analyte recoveries.

\begin{tabular}{|l|c|c|c|}
\hline PAH & $\begin{array}{c}\text { Direct loading } \\
\mathbf{n = 5}\end{array}$ & $\begin{array}{c}\text { GC loading, immediate } \\
\text { analysis, } \mathbf{n}=\mathbf{5}\end{array}$ & $\begin{array}{c}\text { GC loading, stored } \\
\text { overnight, } \mathbf{n}=\mathbf{3}\end{array}$ \\
\hline Phenanthrene & & & 87824 \\
\hline Average peak area & 173635 & 94865 & 29 \\
\hline \% RSD & 6 & 10 & 51 \\
\hline \% recovery & 100 & 55 & \\
\hline
\end{tabular}




\begin{tabular}{|l|c|c|c|}
\hline Pyrene & & & \\
\hline Average peak area & 172019 & 85360 & 92944 \\
\hline$\%$ RSD & 11 & 12 & 5 \\
\hline$\%$ recovery & 100 & 50 & 54 \\
\hline
\end{tabular}

\section{CONCLUSION}

In trace gas analysis, it is important that reliable gas standards can be produced at concentrations which are appropriate both in terms of the relevant applications as well as the method detection limits. In addition, portability and cost are important factors. In the case of the LIF application, a uniform distribution of standard across each of the PDMS tubes was also necessary.

Of the PAHs which were investigated, only the naphthalene diffusion tube had a sufficiently high rate of diffusion to be of experimental use in our studies $\left(\sim 2 \mathrm{ng} . \mathrm{s}^{-1}\right)$. A good correlation was found between the theoretical and the experimental values for the diffusion rate, and steady-state conditions were calculated to be attained in under $5 \mathrm{~min}$. Diffusion tubes are therefore a useful, cost-effective and portable means of generating gas phase standards for the more volatile trace organic air pollutants, but are not suitable for the heavier semi-volatile compounds.

Vapour loading of liquid injected analytes at the FID outlet of a GC via the fraction collection technique developed here proved useful for the PAHs which were less volatile than naphthalene. Repeatability experiments revealed that \% RSDs of $<20 \%$ were possible and recoveries were of the order of 50-60\%, based on the loading of $20 \mathrm{ng}$ of each PAH via a $1 \mu \ell$ injection of a mixed standard. Possible reasons for the low recoveries include losses at the manual injection inlet during trap loading as well as via the mandatory septum purge flow on the split/splitless injector. No significant breakthrough occurred from the multi-channel silicone rubber traps during loading for any of the PAHs investigated under the conditions employed.

The loading of PAHs, specifically phenanthrene and pyrene via this GC based method was therefore shown to be a viable means of loading less volatile analytes onto the silicone rubber traps in the vapour phase. It is also cost effective and allows for the comparison of LIF and GC-MS analyses of the less volatile PAHs. The method offers the additional advantage of loading analytes after capillary chromatographic purification in cases where high purity standards are not readily available and may find application in other spectroscopic studies. 


\section{ACKNOWLEDGEMENTS}

Funding provided by the CSIR, NLC and NRF is gratefully acknowledged.

\section{REFERENCES}

Altshuller AP, Cohen IR (1960) Application of diffusion cells to the production of known concentrations of gaseous hydrocarbons. Anal Chem 32 (7): 802-810

Barratt RS (1981) The preparation of standard gas mixtures. Analyst 106 (1265): 817-849

Danish Environmental Protection Agency (2006) Guidelines on remediation of contaminated sites, Appendix 5.5 Physical and chemical data

Forbes PBC, Rohwer ER (2009) Investigations into a novel method for atmospheric polycyclic aromatic hydrocarbon monitoring. Environ Pollut, doi:10.1016/j.envpol.2009.03.004

Lim Ah Tock MJ (2008) Aroma analysis of alcoholic beverages using multi-channel silicone rubber traps. MTech Thesis, Tshwane University of Technology

Naganowska-Nowak A, Konieczka P, Przyjazny A, Namieśnik J (2005) Development of techniques of generation of gaseous standard mixtures. Crit Rev Anal Chem 35: 31-55

Namieśnik J (1984) Generation of standard gaseous mixtures. J Chromatogr A 300: 79-108

National Metrology Institute of South Africa (2007) Personal communication with A. Botha

Naudé Y, van Aardt M, Rohwer ER (2009) Multi-channel open tubular traps for headspace sampling, gas chromatographic fraction collection and olfactory assessment of milk volatiles. J Chromatogr A, 1216 (14): 2798-2804

Ortner EK, Rohwer ER (1996) Trace analysis of semi-volatile organic air pollutants using thick film silicone rubber traps with capillary gas chromatography. J High Resolut Chromatogr 19: 339344

Wauters E, Van Caeter P, Desmet G, David F, Devos C, Sandra P (2008) Improved accuracy in the determination of polycyclic aromatic hydrocarbons in air using $24 \mathrm{~h}$ sampling on a mixed bed followed by thermal desorption capillary gas chromatography-mass spectrometry. J Chromatogr A 1190: 286-293 